\title{
Boron nitride enhanced polymer/salt hybrid electrolytes for all-solid-state lithium ion batteries
}

\author{
Zhenyu Zhang, Ruiz Gonzalez Antonio, Kwang Leong Choy* \\ UCL Institute for Materials Discovery, University College London, 20 Gordon Street, London, WC1H0AJ, \\ United Kingdom
}

\begin{abstract}
:
Solid state polymer electrolyte is a promising candidate for the next generation of all-solid-state lithium ion batteries due to its advantages of light weight, high stability to electrodes, non-flammable, sufficient mechanical strength to prevent lithium dendrite growth, and low cost. Here, through a facile and cost-effective route, two dimensional boron nitride (BN) is applied as an efficient additive in a polymer/salt hybrid electrolyte, which brings about high ionic conductivity, improved mechanical strength and intimate interfacial contact between the electrolyte and electrodes. A $1 \%$ BN addition into polymer/salt hybrid electrolyte membrane exhibits a high conductivity of $1.82 \times 10^{-3} \mathrm{~S} / \mathrm{cm}$ at room temperature. Indentation test shows the BN modified hybrid electrolyte possesses an enhanced hardness (4.99 MPa) and Young's modulus (0.133 GPa). The 1\% BN modified hybrid electrolyte is demonstrated to effectively suppress the lithium dendrite growth during repeated striping and plating of lithium. As a result, the battery of lithium metal anode paired with $\mathrm{LiFePO}_{4}$ cathode and using the as-fabricated $1 \%$ BN enhanced polymer/salt hybrid electrolyte exhibits improved cycling performance with high Coulombic efficiency (over 98\%).
\end{abstract}

KEYWORDS: solid state electrolyte, polymer, boron nitride, lithium metal anode

\section{Introduction}

Lithium metal is a promising anode of lithium-ion battery (LIB) due to its extremely high theoretical specific capacity (3860 mA h g $\left.{ }^{-1}\right)$, low mass and the lowest negative electrochemical potential (-3.01 $\mathrm{V} \mathrm{vs.} \mathrm{standard}$ hydrogen electrode). However, LIB with lithium metal anode has not been commercialized because it suffers from poor cycling stability, low efficiency and the dangerous Li dendrite growth especially at high current densities.[1] The safety concerns of these batteries using flammable liquid electrolyte (generally $\mathrm{LiPF}_{6}$ in $\mathrm{DC} / \mathrm{DMC}$ ) are that it might cause a short circuit, explosions and fires at high current density operations. In addition, a narrow window 
of operating temperature impedes their further development in the portable devices and electric vehicles. Instead of using liquid electrolyte, solid-state electrolyte (SSE) could greatly improve the safety of LIBs with lithium metal anode, especially it can effectively prevent Li dendrite growth. Moreover, LIBs with SSE and Li metal anode have the potential for achieving higher energy densities than current LIBs.

An ideal candidate of SSE should have the merits of comparable ionic conductivity as present liquid electrolytes, satisfied chemical stability to electrodes as well as ambient environment, wide electrochemical window, high mechanical strength, environmentally benign and low cost. Inorganic SSEs with high ionic conductivities comparable to liquid electrolytes $\left(\sim 10^{-2} \mathrm{~S} \mathrm{~cm}^{-1}\right.$ at room temperature $)$ have been developed.[2,3] For example, sulphide-based SSE $\mathrm{Li}_{9.54} \mathrm{Si}_{1.74} \mathrm{P}_{1.44} \mathrm{~S}_{11.7} \mathrm{Cl}_{0.3}$ exhibits a high room temperature ionic conductivity of $25 \times 10^{-3} \mathrm{~S} \mathrm{~cm}^{-1},[4,5]$ oxide ceramic $\mathrm{SSE} \mathrm{Li}_{7} \mathrm{La}_{3} \mathrm{Zr}_{2} \mathrm{O}_{12}$ shows an ionic conductivity range of $10^{-4}$ to $10^{-3} \mathrm{~S} \mathrm{~cm}^{-1}$ at room temperature which depends on the fabrication process.[6] SSEs are proposed to physically suppress the growth of $\mathrm{Li}$ dendrites and eliminate the irreversible liquid electrolyte decomposition. Therefore, they are expected to have high shear moduli ( $>7 \mathrm{GPa}$, twice of Li metal) and Young's modulus value to promote the application of Li metal anodes.[7] However, there are some critical defects of inorganic SSEs. For example, the high sensitivity of sulphide SSEs to ambient atmosphere greatly limits their preparation; high interfacial resistance from weak physical contact between SSE and electrodes hinders their releasing of the potential for good battery performance. Presently, the synthesis of SSE materials is mainly relying on high temperature sintering under high pressure, which is unfavourable for facile, low-cost thin film SSEs in large scale production.

As an excellent substitution, the polymer/lithium salt system has been used for LIBs with improved safety features and excellent flexibility and processability.[8] For instance, poly(ethylene oxide) (PEO)-based electrolytes that can transfer Li ions by the segmental motion of polymer.[9] However, the low ionic conductivity, poor mechanical and electrochemical stability of PEO-based SSEs limit their practical applications. Generally, the conductivity and mechanical property of the polymer electrolyte could be mutually balanced by varying the content of lithium salt and polymer components, respectively, which is called "salt-in-polymer" or "polymer-insalt" systems. Alternatively, there is a trade-off between conductivity and mechanical property, high conductivity and high strength in one polymer electrolyte are difficult to be achieved simultaneously. Various polymer electrolytes based on cross-linked polymers[10,11] and nanocomposite[12,13,14] utilize hard-soft designs to overcome the challenge between modulus and conductivity. In addition, the application of polymer electrolytes is also impeded by the narrow feasible temperature range (weak thermal stability) because that the elevated temperature (e.g., above $50{ }^{\circ} \mathrm{C}$ ) reduces the mechanical strength. Based on these considerations, poly(vinylidene 
fluoride-co-hexafluoropropylene) (PVDF-HFP) is more promising for solid electrolyte due to its high dielectric constant, low crystallinity, low glass transition temperature $\left(\mathrm{T}_{\mathrm{g}}\right)$, and chemical stability (strong electronwithdrawing C-F bonds).[15]

With excellent mechanical strength, thermal conductivity, chemical stability and electrically insulating properties, two dimensional (2D) boron nitride $(\mathrm{BN})$ is considered as a promising additive in the polymer electrolyte.[16] Particularly, B atoms in BN with Lewis acidic characteristics can interact with other Lewis bases, which are demonstrated to improve the Li-ion transference by attracting the anions in polymer electrolyte.[17] In addition, when $\mathrm{BN}$ is used as an additive in organic ionic liquid electrolytes[18] or a protective coating on $\mathrm{Li}$ metal anodes,[19] it has remarkably suppressed the Li dendrites growth. Therefore, the direct incorporation of 2D BN nanosheets into polymer/lithium salt hybrid electrolyte is expected to enhance both ionic conductivity and mechanical strength, as well as improve the thermal, electrochemical and mechanical stabilities during battery operations.

Although there is a report on "salt-in-polymer" electrolyte enhanced by BN addition, it is fabricated by immersing the composite electrolyte membrane into organic liquid electrolyte in order to absorb the $\mathrm{Li}$-ions, for which the Li-ions concentration might not be precisely controlled, and the process is complicated.[16] In this work, we report a facile solution casting method to fabricate the polymer/salt hybrid electrolyte membrane, which is further enhanced by BN additive. Different concentrations of lithium bis(trifluoromethanesulfonyl)imide (LiTFSI) salt in PVDF-HFP were prepared into membranes, and applied as both electrolyte and separator between two electrodes. It is demonstrated that (1) higher Li-ions concentration leads to higher ionic conductivity of the hybrid electrolyte; (2) $1 \% \mathrm{BN}$ addition can enhance both the mechanical property and ionic conductivity of the composite electrolyte; (3) the $1 \% \mathrm{BN}$ enhanced polymer electrolyte is demonstrated to effectively suppress the lithium dendrite growth, thus inducing improved cycling and rating performance in the LIB of lithium metal anode paired with $\mathrm{LiFePO}_{4}$ cathode.

\section{Materials and experimental}

\subsection{Preparation of the hybrid electrolytes}

All the chemicals were purchased from Sigma-Aldrich. PVDF-HFP pellets $\left(\mathrm{M}_{\mathrm{w}} \sim 455,000\right)$ and LiTFSI with weight ratios of 10/1, 2/1 and 1/1 were dissolved in dimethylformamide (DMF) and acetone (2:1 v/v). After stirred at $70{ }^{\circ} \mathrm{C}$ for 24 hours, the clear viscous solution was casted onto glass substrate and controlled to $500 \mu \mathrm{m}$ thick by a doctor-blade, which was then dried in vacuum at $70{ }^{\circ} \mathrm{C}$ for 24 hours. A white film was formed on the glass and 
could be easily peeled off to form a free-standing membrane. The dried membrane had a thickness of $\sim 150 \mu \mathrm{m}$. For the BN added membranes, different amounts of BN nanosheet powder $(\sim 1 \mu \mathrm{m})(1 \%$ and $10 \% v s$. the total weight of PVDF-HFP and LiTFSI) were added into the above solution and stirred for another 2 hours. The remaining procedures were same as the preparation of membranes without $\mathrm{BN}$.

\subsection{Materials characterization}

The morphology of the hybrid electrolyte and lithium surface after cycling were observed by scanning electron microscope (SEM) (ZEISS EVO® LS15). X-ray diffraction (XRD) (Philips/PANalytical X'Pert PRO, with a Cu $\mathrm{K}_{\alpha}$ radiation source operated at $40 \mathrm{kV}$ and $40 \mathrm{~mA}$, tested with a scan rate of $0.05^{\circ} \mathrm{s}^{-1}$ and 2 theta from $10^{\circ}$ to $70^{\circ}$ ) and Fourier Transform Infrared Spectroscopy (FTIR) (Perkin Elmer Spectrum Two, tested with a wavenumber of from 400 to $4000 \mathrm{~cm}^{-1}$ ) were used to check the existence of BN. Thermogravimetric analyser (TGA) (PerkinElmer TGA 4000 System) curves of the samples were obtained under air flowing at a heating rate of $10{ }^{\circ} \mathrm{C} \mathrm{min}^{-1}$ from 30 to $700{ }^{\circ} \mathrm{C}$. The mechanical properties of the samples were tested with a depth $v s$. load experiment using NanoTest Vantage (using a diamond Berkovich tip). The loading force was up to $20 \mathrm{mN}$ and the contact depth was up to $10,000 \mathrm{~nm}$. Hysteresis curves were obtained based on $10 \times 10$ points within an area of $300 \times 300 \mu \mathrm{m}^{2}$, which gave the average hardness and reduced modulus results of the 100 points by the NanoTest Vantage software. The BET surface area and pore-size distribution analysis were carried out by NOVA Touch LX4 Quantachrome gas sorption analyser.

\subsection{Electrochemical measurements}

As-prepared polymer/salt hybrid electrolyte membrane was cut into disks with diameters of 13 or $16 \mathrm{~mm}$. CR2032 coin cell was assembled in the Argon filled glove box (LABmaster pro SP). Symmetric cell was composed of two lithium metal pellets with a diameter of $13 \mathrm{~mm}$, and the as prepared polymer/hybrid electrolytes (16 mm). Asymmetric cells of Li/hybrid electrolyte/stainless steel with the three hybrid electrolytes were assembled and linear sweep voltammetry (LSV) were conducted between $3.0-5.5 \mathrm{~V}$ with a scan rate of $1 \mathrm{mV} \mathrm{s}^{-1}$. Asymmetric cells were also assembled by using lithium metal anode, liquid or the polymer/salt hybrid electrolytes, and $\mathrm{LiFePO}_{4}$ cathode material on $\mathrm{Al}$ foil (single side, $13 \mathrm{~mm}$ in diameter, $\sim 18 \mathrm{mg}$ active material per pellet (13.6 $\mathrm{mg} \mathrm{cm}{ }^{-2}$ ), purchased from MTI corporation). In addition, liquid electrolyte (lithium hexafluorophosphate solution in ethylene carbonate and dimethyl carbonate, i.e., 1.0 $\mathrm{M} \mathrm{LiPF}_{6}$ in $\left.\mathrm{EC} / \mathrm{DMC}=50 / 50(\mathrm{v} / \mathrm{v})\right)$ was also used for comparison in the test. All the electrochemical impedance spectrum (EIS) of electrolyte membrane $(13 \mathrm{~mm}$ diameter) with two stainless steel plates, symmetric coin cells, asymmetric coin cells were tested and analysed by 
Gamry Interface 1010E. The EIS spectra were obtained with oscillation amplitude of $10 \mathrm{mV}$ over the frequency range from $1 \mathrm{MHz}$ to $0.1 \mathrm{~Hz}$ at room temperature $\left(21.0{ }^{\circ} \mathrm{C}\right)$ or controlled from 20 to $100{ }^{\circ} \mathrm{C}$ inside an oven. The cycling performance of different batteries were measured by Maccor (Model 4300M) at ambient temperature. The galvanostatic cycling of the symmetric batteries were tested at a current density of $0.1 \mathrm{~mA} \mathrm{~cm}^{-1}, 0.1 \mathrm{mAh} \mathrm{cm}^{-1}$ for each charge or discharge. The cycling and rating performance of asymmetric cells were measured between 2.5 and $4.4 \mathrm{~V}$ with $0.1 \mathrm{C}-1 \mathrm{C}\left(1 \mathrm{C}=170 \mathrm{~mA} \mathrm{~g}^{-1}\right)$ current density.

\section{Results and discussion}

\subsection{Characterization of Materials}

The polymer/salt hybrid electrolyte membrane was prepared via a facile solution casting method, the photos of as obtained membrane is presented in Fig. S1. The white membrane has a uniform thickness of about $168 \mu \mathrm{m}$, and the process can be scale-up for mass production. As a note in advance, the hybrid electrolyte membranes of different polymer/salt weight ratios and different mass of BN addition have the similar thickness $(\sim 150 \mu \mathrm{m})$ and appearance. The polymer/salt hybrid electrolyte composed of PVDF-HFP and LiTFSI with different weight ratios were first prepared and their ionic conductivities were compared. As demonstrated, the resistance from EIS spectra (Nyquist plot) decreased distinctly as the Li-ion concentration increase (Fig. S2). The bulk resistance of the polymer/salt hybrid electrolyte membranes $(1 / 1,2 / 1$ and 10/1) are $29 \Omega, 49 \Omega$, and $10000 \Omega$, respectively. Thus the conductivities were calculated to be $3.92 \times 10^{-4}, 2.32 \times 10^{-4}$, and $1.13 \times 10^{-6} \mathrm{~S} / \mathrm{cm}$, respectively, based on their thickness $(150 \mu \mathrm{m})$ and contact area $($ diameter $=13 \mathrm{~mm})$. In the following text, the polymer/salt hybrid electrolyte membrane is denoted to $1 / 1$ sample, which is used for further investigation of the properties of $\mathrm{BN}$ modified electrolytes.

SEM images of the polymer/salt hybrid electrolytes without $\mathrm{BN}$, with $1 \% \mathrm{BN}$ and $10 \% \mathrm{BN}$ are presented in Fig. 1. As shown in Fig. 1a-b, PVDF-HFP with dissolved LiTFSI salt forms a sphere structure with a uniform diameter of about $2 \mu \mathrm{m}$. The spheres are connected to be a network of free-standing membrane, which has a large proportion of pores among the spheres. The formation of the spherical grain structure is attributed to surface tension of the in-situ aggregated polymer chains during the evaporation of the solvent.[20] By contrast, with $1 \%$ $\mathrm{BN}$ addition, a denser and more connective membrane is produced, with a smaller and non-uniform size of the polymer/salt particle. Due to the small size $(\sim 1 \mu \mathrm{m}$, Fig. S3) and $2 \mathrm{D}$ characteristic of BN nanosheets, the BN component cannot be observed explicitly. A magnified picture of inset in Fig. 1d shows some fine dots on the surface of the merged polymer materials, which can be attributed to the existence of BN nanosheets. 
Comparatively, $10 \% \mathrm{BN}$ addition induces a more uniform membrane with flat surface and lowest porosity, as exhibited in Fig. 1e-f. The particle size of polymer/salt is much reduced $(<1 \mu \mathrm{m})$, which are well connected by the BN nanosheets. Fig. S4 gives the EDS mapping of the $1 \% \mathrm{BN}$ modified hybrid electrolyte, which presents a uniformly distributed boron and nitrogen elements in the carbon and fluorine matrix of PVDF-HFP, and sulphur of LiTFSI.

In addition to the SEM images, we also conducted the $\mathrm{N}_{2}$ adsorption-desorption measurements to analyse the microstructure of the hybrid electrolytes. As given in Fig. S5, the surface area of the hybrid electrolytes without $\mathrm{BN}$, with $1 \%$ and $10 \% \mathrm{BN}$ are 5.065, 0.373, and $1.004 \mathrm{~m}^{2} \mathrm{~g}^{-1}$, respectively, which agrees well with the SEM images. The hybrid electrolyte without BN has a network structure of linked spheres, inducing its much larger surface area and $\mathrm{N}_{2}$ adsorption volume. The $1 \% \mathrm{BN}$ modified hybrid electrolyte has the lowest surface area and $\mathrm{N}_{2}$ adsorption volume, implying the highest density. In addition, the hybrid electrolyte with BN has wider poresize distribution range than that without $\mathrm{BN}$, due to the heterogeneous material distribution in the hybrid electrolyte with BN addition. To summarize, the adding of BN nanosheets reshaped the microstructure of PVDF$\mathrm{HFP} /$ salt hybrid electrolyte, making it more connective and a denser free-standing membrane.
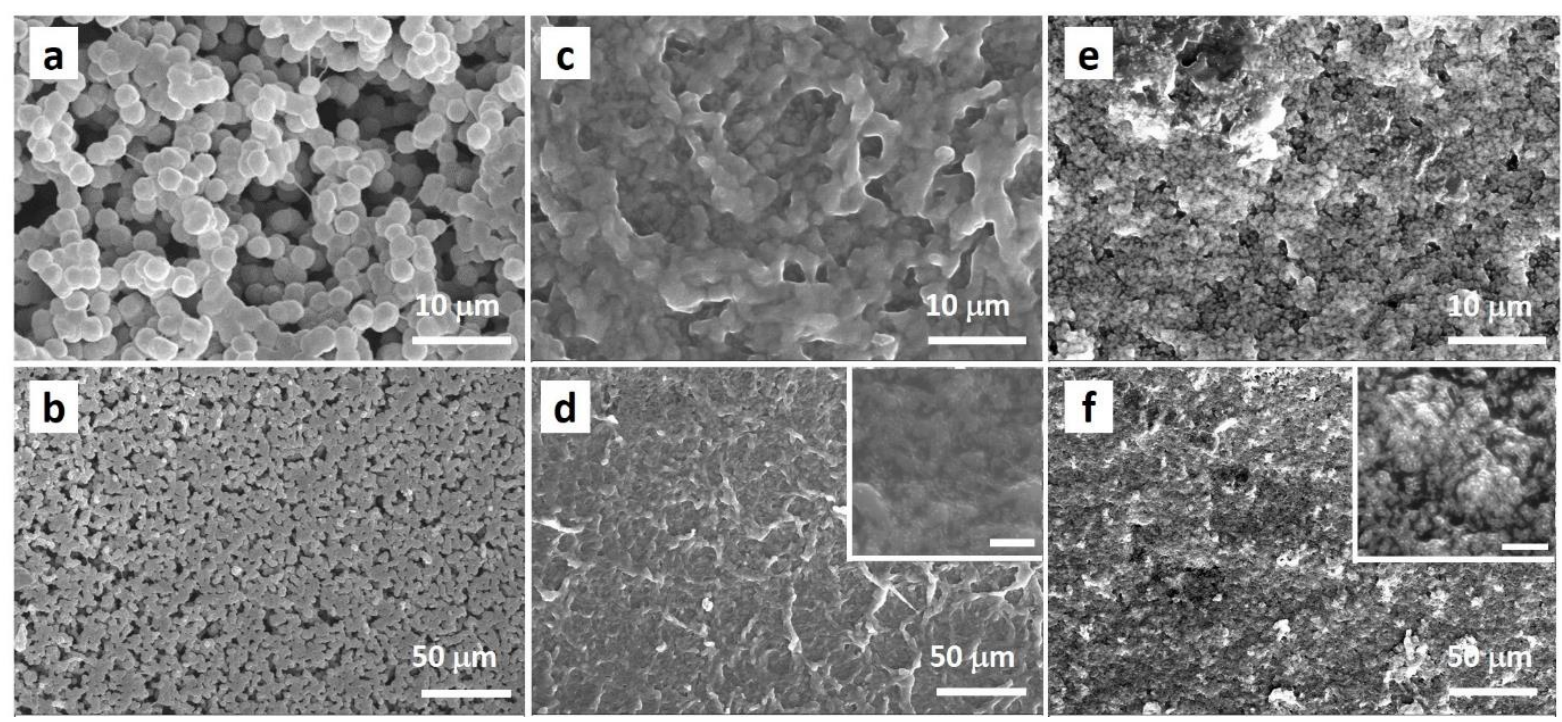

Fig. 1. SEM images of the PVDF-HFP/LiTFSI hybrid electrolyte (a, b) without BN, (c, d) with $1 \% \mathrm{BN}$ and (e, f) $10 \% \mathrm{BN}$ addition. Scale bars in the inset of (d) and (f) are $2 \mu \mathrm{m}$.

The chemical compositions of polymaer/salt hybrid electrolytes with different $\mathrm{BN}$ addition percentages were analysed by FTIR and XRD. The FTIR spectra (Fig. 2a and Fig. S6) of the hybrid electrolytes without BN, with $1 \%$ and $10 \% \mathrm{BN}$ addition show almost overlapped profiles, most of the transmittance peaks are attributed to the 
PVDF-HFP (including 760, 850, 872, 973, 1060, 1146, 1292, 1381, $1404 \mathrm{~cm}^{-1}$, corresponding to $\mathrm{CF}_{3}$ group, $\mathrm{CH}_{2}$ rocking, $\mathrm{CH}_{2}$ wagging of the vinylidene, out of plane $\mathrm{C}-\mathrm{H}$ bending, symmetric $\mathrm{C}-\mathrm{F}$ stretching, $\mathrm{CF}_{2}$ stretching, symmetrical stretching $\mathrm{CF}_{3}, \mathrm{CH}_{2}$ wagging and scissoring vibration of vinylidene) and LiTFSI (including 1732, $\left.1651,1441,1350,1182,1055,1022,788,739 \mathrm{~cm}^{-1}\right) .[21,22,23]$ The peaks from PVDF-HFP and LiTFSI almost cover all the peaks, and some of them are overlapped and intensified by each other. The peaks belong to h-BN are centred at 1370 and $810 \mathrm{~cm}^{-1}$, both of which have a large peak width (the range are $1520-1220$, and $850-770 \mathrm{~cm}^{-}$ 1, respectively).[24] As shown clearly in Fig. 2a, the peaks belong to PVDF-HFP and LiTFSI in the two ranges are all intensified by the wide BN peaks, and getting more intense as the increase of BN content. XRD pattern (Fig. 2b) shows the amorphous phase of the polymer material, which is favourable for contributing to a higher ionic conductivity.[25] The intensity of the peaks at $26.3^{\circ}$ and $41.1^{\circ}$, which are indexed to $h$-BN $((002)$ and (100) plane), increases as more $\mathrm{BN}$ component is added into the polymer/salt hybrid electrolyte. In addition, the diffraction peaks belong to PVDF-HFP $\left(18.4^{\circ}, 20.8^{\circ}, 26.6^{\circ}\right.$ corresponding to the PVDF $\alpha$-phase, $\beta$-phase and $\gamma$ phase diffraction) are not showing a decreasing trend as the increasing of $\mathrm{BN}$ content, implying that the crystallinity of polymer is not lowered.[26] Thermal stability of the polymer/salt hybrid electrolytes with different BN addition is compared in Fig. 2c. Without BN, the TGA curve of PVDF-HFP/LiTFSI membrane exhibits a fast weight loss as the temperature increases from $30{ }^{\circ} \mathrm{C}$, only $80 \%$ of the initial weight remained at $300{ }^{\circ} \mathrm{C}$. Due to the decomposition temperature of LiTFSI and PVDF-HFP in air are about $380{ }^{\circ} \mathrm{C}$ [27] and $475{ }^{\circ} \mathrm{C}$,[28-30] respectively, the weight loss in this period is partially attributed to the evaporated of residual solvent after the drying process of hybrid electrolyte membrane preparation. The obvious weight losses after $300{ }^{\circ} \mathrm{C}$ and $400{ }^{\circ} \mathrm{C}$ in the TGA curve of the PVDF-HFP/LiTFSI hybrid electrolyte are believed to be due to the decomposition of LiTFSI and PVDF-HFP, and finally the weight was close to zero after $600{ }^{\circ} \mathrm{C}$. Apparently, the decomposition of hybrid electrolyte without $\mathrm{BN}$ is faster than that of with $1 \%$ and $10 \% \mathrm{BN}$, both of which kept stable until $150{ }^{\circ} \mathrm{C}$, then gradually dropped to $\sim 90 \%$ of the initial weight at $300{ }^{\circ} \mathrm{C}$. After decomposed at higher temperatures, the final weights of the two samples were about $1 \%$ and $10 \%$ of the initial weight, respectively. Similar to the TGA results, it is further confirmed by a set of photographs as shown in Fig. S7, the three membranes were heated to 150, 300, and $350{ }^{\circ} \mathrm{C}$ in air, that $\mathrm{BN}$ addition is favourable for improving the thermal stability of the polymer/salt hybrid electrolyte.

Mechanical properties are significant for solid electrolyte membranes used in LIBs, especially those with lithium metal anodes. The solid electrolyte membrane is supposed to be strong enough (have high Young's modulus) to suppress the lithium dendrite growth during the repeated lithium metal striping/plating on the surface 
of electrodes. Most of the similar works tested the mechanical properties by employing tensile measurement, which gives the strain $v s$. stress curve and the tensile strength.[11,16,31,32] Here the mechanical properties of the polymer/salt hybrid electrolytes were tested by indentation experiment with a diamond Berkovich tip, producing a depth vs. load hysteresis (Fig. S8) and the calculated hardness and reduced modulus, which might be a more analogous process for simulating the dendrite growth in batteries than tensile experiment. As shown in Fig. 2d, both hardness and reduced modulus increase as $\mathrm{BN}$ addition percentage increases. The hardness of the electrolyte without $\mathrm{BN}$, with $1 \%$ and $10 \% \mathrm{BN}$ are $2.37,4.99$ and $8.55 \mathrm{MPa}$, respectively, and the reduced modulus (approximately equal to Young' modulus as explained in Fig. S8) are 0.062, 0.133, and 0.206 GPa, respectively. The $10 \%$ BN modified electrolyte has the highest hardness and reduced modulus, however the values are not enhanced linearly with the $\mathrm{BN}$ proportion. As compared with polymer/salt hybrid electrolyte without $\mathrm{BN}, 1 \% \mathrm{BN}$ addition doubles both values, and the Young's modulus of $0.133 \mathrm{GPa}$ is higher than that of the reported values in similar works by tensile measurement.[11,16,31,32]
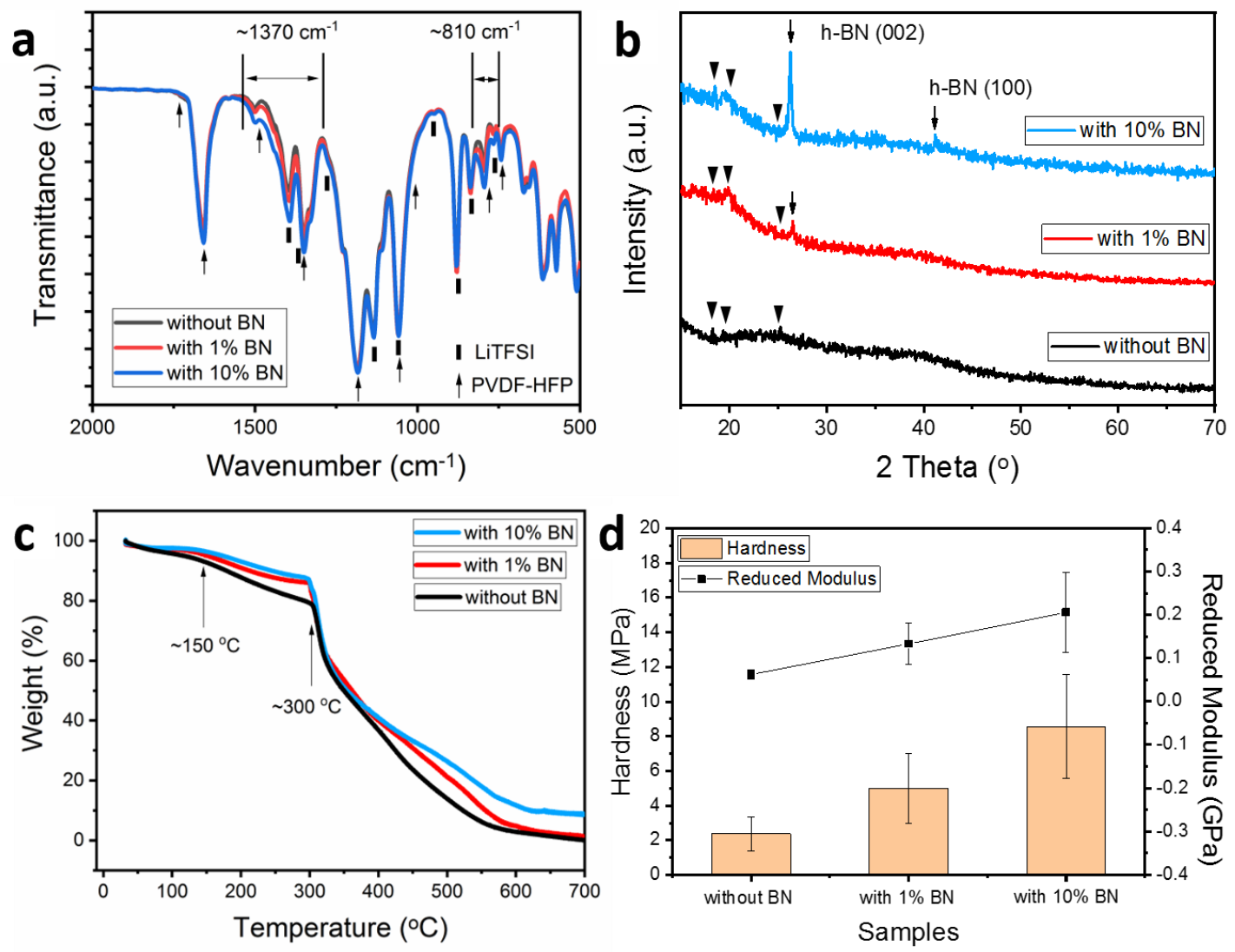

Fig. 2. (a) FTIR spectra, (b) XRD patterns (black triangles are indexed to the diffraction peaks of PVDF-HFP), (c) TGA curves, (d) mechanical test results of the polymer/salt hybrid electrolytes without BN, with $1 \% \mathrm{BN}$ and with $10 \% \mathrm{BN}$. 


\subsection{Electrochemical test of the hybrid electrolytes}

The ionic conductivity of the polymer/salt hybrid electrolytes with different proportions of BN additions were obtained from the EIS tests conducted at different temperatures with two stainless steel blocking electrodes. Fig. 3a presents the magnified Nyquist plots of the three polymer/salt hybrid electrolytes at room temperature. The intercept on the $\mathrm{X}$ axis of the electrolytes without $\mathrm{BN}$, with $1 \%$ and $10 \% \mathrm{BN}$ are $28.29,10.40,20.04 \Omega$, respectively, which is an indication of their intrinsic resistance. The ionic conductivities of the electrolytes were calculated based on the thickness, effective electrolyte-electrode contact area, and the bulk resistance fitted by the equivalent circuit as shown in Fig. 3a, where $R_{e}$ is the bulk resistance of electrolyte, $R_{i}$ and $Q_{i}$ are the contact interfacial resistance and constant phase element (CPE) between electrolyte and electrode, $\mathrm{W}$ is the Warburg diffusion resistance. The calculated ionic conductivities at room temperature of the three samples are $6.69 \times 10^{-4}$, $1.82 \times 10^{-3}$, and $9.45 \times 10^{-4} \mathrm{~S} \mathrm{~cm}^{-1}$, respectively. These values are comparable to that of PVDF-HFP with $50 \% 1 \mathrm{M}$ $\mathrm{LiPF}_{6}$ in EC/PC gel-type polymer electrolyte, in which the content of solvent plays important rule for the enhanced ionic conductivity.[33] According to the TGA results, since 10\% and 20\% residual solvent (mainly DMF due to its higher boiling point than acetone) in the hybrid electrolytes with and without BN addition according to the TGA results, we consider that the remained solvent in the electrolytes would also contribute to the high ionic conductivity. By elevating the temperatures to $40,60,80$ and $100{ }^{\circ} \mathrm{C}$, the conductivities of the three electrolytes were also obtained and given in Table S1 and Fig. S9. The 1\% BN added polymer/salt hybrid electrolyte reached an ionic conductivity of $3.47 \times 10^{-3} \mathrm{~S} \mathrm{~cm}^{-1}$ at $100^{\circ} \mathrm{C}$. Fig. $3 \mathrm{~b}$ shows the Arrhenius plot of the three electrolytes. The activation energy $E_{a}$ was calculated according to the classical Arrhenius equation $\sigma(T)=A \exp \left(-E_{a} / R T\right)$, where $T$ is the absolute temperature and $\mathrm{A}$ is a pre-exponential factor. With the addition of $1 \% \mathrm{BN}, \mathrm{E}_{\mathrm{a}}$ for the polymer/salt hybrid electrolyte is $0.076 \mathrm{eV}$, which is lower than that of the electrolyte without $\mathrm{BN}(0.124 \mathrm{eV})$ and with $10 \%$ $\mathrm{BN}(0.123 \mathrm{eV})$. A lower $\mathrm{E}_{\mathrm{a}}$ indicates faster ionic migration, $[28,34]$ therefore, with $1 \% \mathrm{BN}$ addition, the ion mobility is superior to the counterparts of without $\mathrm{BN}$ and with $10 \% \mathrm{BN}$.

The EIS of $2 \%$ BN added hybrid electrolyte was also tested, which confirmed that hybrid electrolyte with $1 \%$ BN possesses the highest ionic conductivity among the prepared samples (Fig. S10). Generally, the ionic conductivity of polymer-based electrolytes are improved by adding fillers like $\mathrm{SiO}_{2}$ or $\mathrm{Al}_{2} \mathrm{O}_{3}$ nanoparticles, due to the reduced crystallization of the polymer materials. $[35,36]$ However, in this work, the diffraction peaks of PVDF-HFP (Fig. 2b) are not showing a decreasing trend as the increasing of BN content. Since the ionic transfer mainly takes place inside the bulk rather than the surface of the Li ions contained polymer materials, higher density and less pores of polymer induces easier ion mobility. By adding $1 \% \mathrm{BN}$ in the polymer electrolyte, it shows 
increased density, decreased surface area and porosity (Fig. 1 and S5), and thus superior ionic conductivity. However, too much insulated BN (10\%) in the polymer leads to worse ionic transport ability. It is reported that as an additive in polymer based electrolyte, BN can work as a Lewis acid to trap the anions of the electrolyte, leading to improved Li-ion transference.[16] Therefore, the ionic conductivity enhancement by the BN addition is ascribed to both the increased density and special chemical property of BN. Furthermore, the EIS of the four hybrid electrolytes at room temperature after stored in a dry box after 6 months are compared (Fig. S10) with the freshly prepared hybrid electrolytes, the ionic conductivity for the hybrid electrolytes without BN, with $1 \%, 2 \%$ and $10 \% \mathrm{BN}$ are decreased to $3.88 \times 10^{-5}, 4.33 \times 10^{-4}, 3.51 \times 10^{-4}$, and $2.82 \times 10^{-4} \mathrm{~S} \mathrm{~cm}^{-1}$, which are $17.25,4.2,3.8$, and 3.35 times lower, respectively. Therefore, the $\mathrm{BN}$ addition is demonstrated to have the ability of preventing conductivity ageing after long term storage for the hybrid electrolyte. The room temperature ionic conductivity of the hybrid electrolyte with $1 \% \mathrm{BN}$ is among the best values of the polymer-based hybrid electrolyte, as compared with other reported works in Table S2.

Fig. 3c presents the linear sweep voltammetry (LSV) of the asymmetric cells of Li/hybrid electrolyte/stainless steel with the three hybrid electrolytes. The obvious degradation (current of $0.01 \mathrm{~mA}$ ) occurs till $4.7 \mathrm{~V}, 4.8 \mathrm{~V}$ and 5.0 $\mathrm{V}$ versus $\mathrm{Li}^{+} / \mathrm{Li}$ for the hybrid electrolytes without $\mathrm{BN}$, with $1 \%$ and $10 \% \mathrm{BN}$, respectively. The increased oxidation potential with the increase of BN content in the hybrid electrolyte implies that $\mathrm{BN}$ addition induces larger electrochemical stability window of hybrid electrolyte by lowering the extent of anion oxidation at a high potential.[16] This result indicates that the hybrid electrolyte membrane with BN addition can satisfy the requirements of most high-voltage lithium batteries.[37]

Symmetric cells using two lithium metal electrodes with the as-fabricated electrolytes were assembled to investigate the interfacial properties of the hybrid electrolytes against lithium metal. Fig. 3d displays the Nyquist plot of the symmetric cells with the polymer/salt hybrid electrolytes without BN, with $1 \%$ and $10 \%$ BN addition. Symmetric cell with liquid electrolyte and glass fibre separator is also used for comparison, which exhibits very small resistance (including resistance of the solution and the interfacial resistance with electrode) due to high ionic conductivity and good wettability. The EIS spectra could be equal to the circuit shown in the inset, which is divided into an ohmic resistance $\left(\mathrm{R}_{\mathrm{e}}\right)$ and $\mathrm{CPE}\left(\mathrm{Q}_{\mathrm{e}}\right)$ of electrolyte at high frequency, and interfacial resistance $\left(\mathrm{R}_{\mathrm{i}}\right)$ and $\mathrm{CPE}\left(\mathrm{Q}_{\mathrm{i}}\right)$ of the contact layer between electrolyte and electrode at low frequency. The large semicircles of the three electrolytes are attributed to the interfacial resistance.[38,39] The fitted values for the interfacial resistance of each interface (note there are two equal interfaces in a cell) are $255 \Omega, 114 \Omega$ and $156 \Omega$ for the polymer/salt 
hybrid electrolytes without $\mathrm{BN}$, with $1 \% \mathrm{BN}$ and with $10 \% \mathrm{BN}$, respectively. Therefore, $1 \% \mathrm{BN}$ addition into the polymer/salt hybrid electrolyte contributes to a lowest contact resistance between electrolyte and electrode.

The mechanical stability of solid electrolyte membrane during the electrochemical process is important for maintaining the long cycle life of batteries. During charge and discharge processes at a constant current, lithium ions are repeatedly stripping/plating on the surface of lithium metal electrodes. The non-uniform stripping/plating of metal lithium would induce the lithium dendrite growth, until the electrolyte membrane be impaled and the battery be short circuited. As presented in Fig. 3e, the symmetric cells with the three electrolytes were operated at a current density of $0.1 \mathrm{~mA} \mathrm{~cm}-1$, and each charge or discharge process lasts for $1 \mathrm{~h}$, which contributes to a capacitance of $0.1 \mathrm{~mA} \mathrm{~h} \mathrm{~cm}{ }^{-1}$. The cells of polymer/salt hybrid electrolytes without $\mathrm{BN}$ and with $10 \% \mathrm{BN}$ short circuited after $77 \mathrm{~h}$ and $193 \mathrm{~h}$, respectively. By contrast, the symmetric cell of polymer/salt hybrid electrolyte with $1 \% \mathrm{BN}$ was more stable and cycled for $300 \mathrm{~h}$ without short-circuiting. In addition, at $50 \mathrm{~h}$, the cell with $1 \% \mathrm{BN}$ electrolyte exhibits a significantly lower overpotential $(\sim 0.15 \mathrm{~V})$ than that of $10 \% \mathrm{BN}$ electrolyte $(\sim 0.38 \mathrm{~V})$, and electrolyte without $\mathrm{BN}(\sim 0.25 \mathrm{~V})$, agreeing with the measured highest ionic conductivity of $1 \% \mathrm{BN}$ electrolyte.[34] The inset magnifies the three cycles between $286 \mathrm{~h}$ and $289 \mathrm{~h}$, which clearly shows the stable voltage profile after a long cycle time. To further confirm the ability to suppress the lithium dendrite growth of the three electrolytes, cells were stopped at $50 \mathrm{~h}$, disassembled, and the surface morphology of lithium metal was examined by SEM, as presented in Fig. 4. As compared with the pristine smooth Li metal surface (Fig. 4a-b), there are massive cracks and protuberances on the Li electrode worked with the polymer/salt hybrid electrolyte without $\mathrm{BN}$ after $50 \mathrm{~h}$ galvanostatic striping/plating (Fig. 4c-d). On the contrary, the Li electrode from the cell with $1 \% \mathrm{BN}$ in the electrolyte only shows a few shallow pits (Fig. 4e-f). Comparatively, the Li surface of 10\% BN added polymer electrolyte shows more pits and higher roughness (Fig. 4g-h). This might be caused by the lowered ionic conductivity and non-uniform distribution of the compositions due to too much BN. The Li stripping/plating at a higher current density of $1 \mathrm{~mA} \mathrm{~cm}^{-2}$ is given in Fig. S11, in which the hybrid electrolyte without BN and with $10 \%$ BN show shorter cycle life than that of hybrid electrolyte with $1 \%$ BN. However, the symmetric cells with hybrid electrolyte with $1 \% \mathrm{BN}$ shows gradually increased voltage, indicating the worse cycling stability than its performance at lower current density. In summary, the addition of $1 \% \mathrm{BN}$ into the polymer/salt hybrid electrolyte is favourable for preventing lithium dendritic growth (Fig. 4i), too much BN (e.g., 10\%) and without BN added polymer electrolytes would lead to uneven striping/plating of Li metal on the electrodes. 

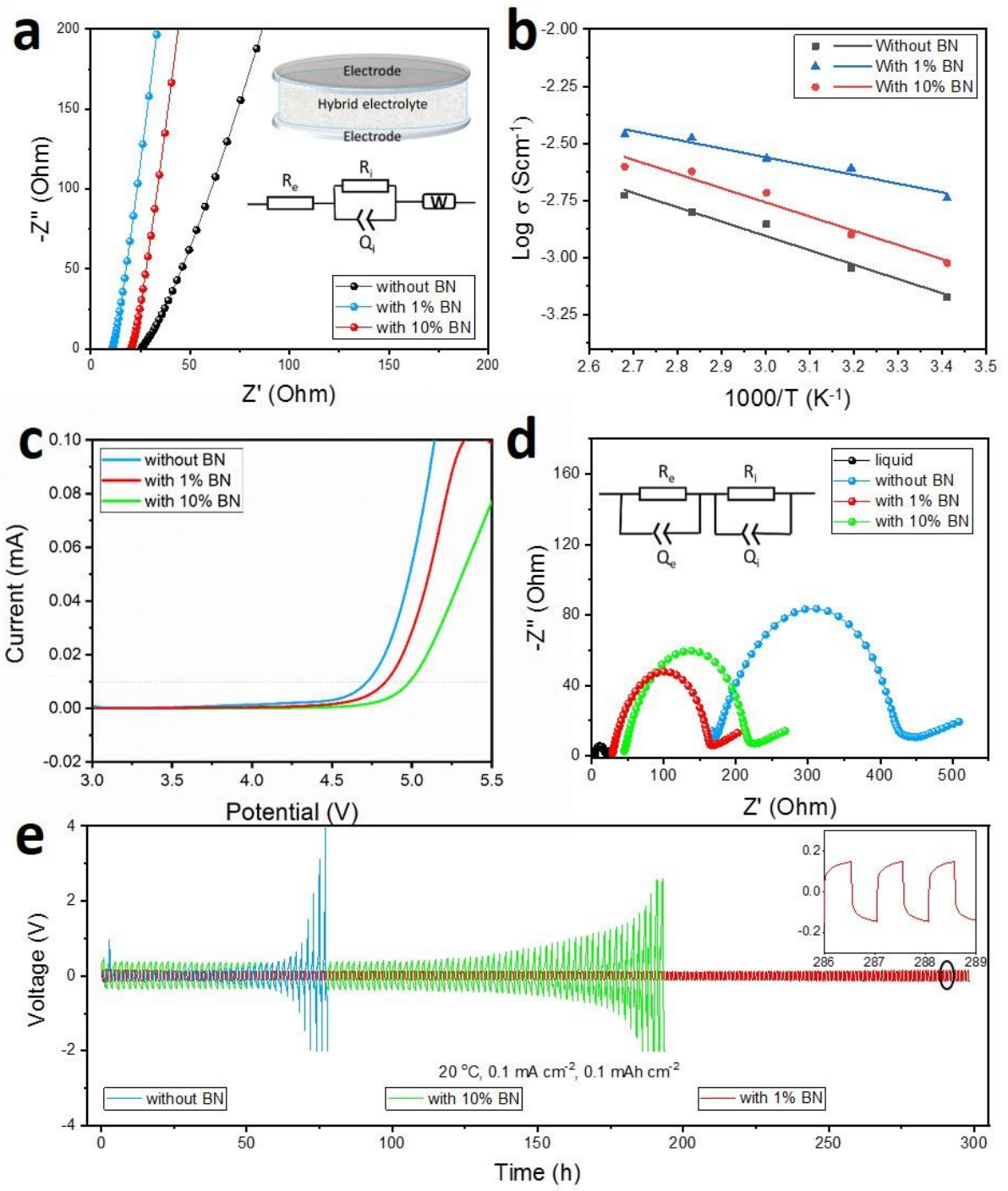

Fig. 3. (a) EIS spectra (Nyquist plot) of the polymer/salt hybrid electrolytes without BN, with $1 \% \mathrm{BN}$ and with $10 \% \mathrm{BN}$ at room temperature. Inset is the equivalent circuit. (b) Arrhenius plot of the three electrolytes. (c) LSV of the Li/hybrid electrolyte/stainless steel cells in the potential range of 3.0-5.5 V with a scan rate of $1 \mathrm{mV} \mathrm{s}^{-1}$. (d) EIS spectra (Inset is the equivalent circuit) of the symmetric cells with the liquid and three hybrid electrolytes. (e) lithium striping/plating cycling at a current density of $0.1 \mathrm{~mA} \mathrm{~cm}{ }^{-2}$ of the symmetric cells using the three electrolytes. Inset is the magnified image of the three cycles between 286 and $289 \mathrm{~h}$ of the polymer/salt hybrid electrolyte with $1 \% \mathrm{BN}$. 

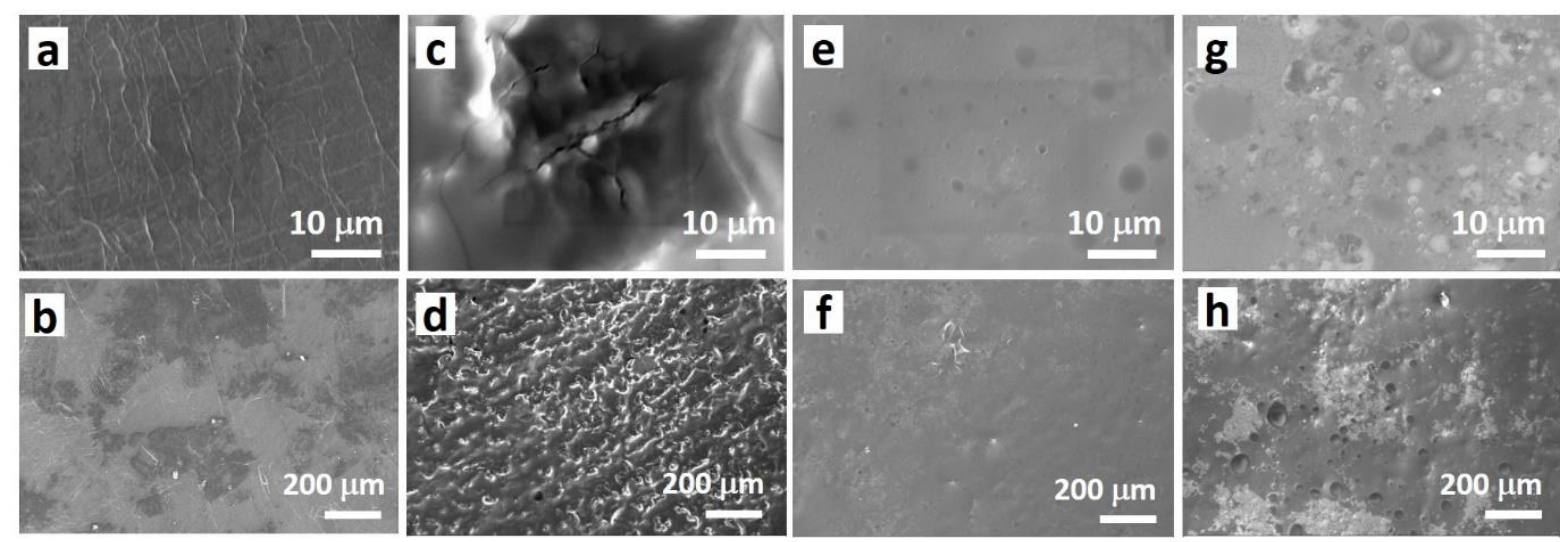

i

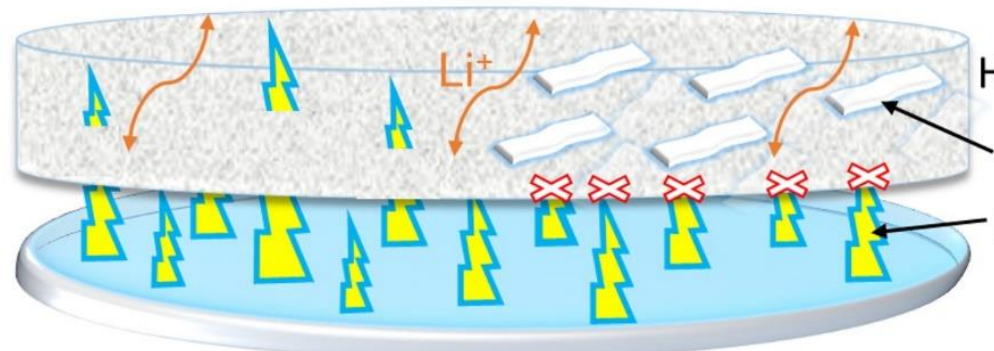

Hybrid electrolyte

BN nanosheets

Lithium dendrite

Lithium anode

Fig. 4. SEM images of the Li metal surface after the galvanostatic cycling for $50 \mathrm{~h}$ of the symmetric batteries. (a, b) Pristine Li metal. Li metal electrodes from the cells of electrolyte without BN (c, d), with $1 \%$ BN (e, f) and with $10 \% \mathrm{BN}(\mathrm{g}, \mathrm{h})$. (i) A schematic illustration of the role of BN in the polymer/salt hybrid electrolyte in suppressing the lithium dendrite growth during lithium striping/plating.

\subsection{Electrochemical tests of asymmetric battery}

To evaluate the electrochemical performance of the polymer/salt hybrid electrolytes, coin cells (asymmetric) with lithium metal anode, $\mathrm{LiFePO}_{4}$ cathode on $\mathrm{Al}$ foil current collector and as-prepared electrolyte membranes were assembled and tested. Fig. 5a shows the Nyquist plot of the freshly assembled asymmetric cells. The battery with liquid electrolyte and glass fibre separator shows lowest overall resistance $(18.5 \Omega)$. For the polymer/salt hybrid electrolytes, the EIS results can be fitted by the equivalent circuit as shown in the inset, which includes the electrolyte resistance $\left(\mathrm{R}_{\mathrm{e}}\right)$, interface resistance $\left(\mathrm{R}_{\mathrm{i}}\right)$ and charge transfer resistance $\left(\mathrm{R}_{\mathrm{ct}}\right)$. It can be apparently extracted that $\mathrm{BN}$ addition into the polymer/salt hybrid electrolyte can reduce the resistance of all elements. In addition, $1 \% \mathrm{BN}$ electrolyte exhibits a lower overall resistance than that of $10 \% \mathrm{BN}$ electrolyte. Fig. $5 \mathrm{~b}$ shows the rating performance of the three batteries using polymer/salt hybrid electrolytes at current densities of $0.1 \mathrm{C}, 0.2 \mathrm{C}$, $0.5 \mathrm{C}$ and $1 \mathrm{C}$, in the voltage range of $2.5 \mathrm{~V}$ and $4.4 \mathrm{~V}$. The battery with liquid electrolyte shows higher capacities at high current densities due to the good contact between electrode material and the liquid electrolyte. Among the three polymer/salt hybrid electrolytes, the one with $1 \% \mathrm{BN}$ shows a superior rate capability. For this battery, the $\mathrm{LiFePO}_{4}$ cathode delivers a charge capacity of $113.5 \mathrm{~mA} \mathrm{~h} \mathrm{~g}^{-1}$ at a current density of $1 \mathrm{C}\left(170 \mathrm{~mA} \mathrm{~g}^{-1}\right)$, mainly due 
to the higher ionic conductivity than other hybrid electrolytes. However, at high current density, the capacity of the battery with $1 \% \mathrm{BN}$ added electrolyte is lower than that of liquid electrolyte, indicating its worse capability than liquid electrolyte. Polarization is occurred at high current densities due to larger potential gap between oxidation and reduction reactions is appearing, which is shown in Fig. S12.

Fig. 5c presents the cycling performance of the batteries in the voltage range of 2.5 and $4.4 \mathrm{~V}$ at $0.2 \mathrm{C}$ within 50 cycles (the first charge/discharge cycle is operated at $0.02 \mathrm{C}$ rate). The battery with liquid electrolyte delivers a capacity of $112.6 \mathrm{~mA} \mathrm{~h} \mathrm{~g}^{-1}$ after 50 cycles, with a capacity retention of $\sim 80 \%$. Similarly, the battery of the polymer/salt hybrid electrolyte with $1 \% \mathrm{BN}$ exhibits capacities of over $150 \mathrm{~mA} \mathrm{~h} \mathrm{~g}^{-1}$ at the first three cycles, and after a gradually decrease, it remains at $116.0 \mathrm{~mA} \mathrm{~h} \mathrm{~g}^{-1}$ at the $50^{\text {th }}$ cycle. The capacity retention is even slightly higher than the battery with liquid electrolyte. The battery with $10 \% \mathrm{BN}$ in the hybrid electrolyte shows similar reversible capacities to the former two counterparts at the beginning, but the capacity drop is accelerated after $25^{\text {th }}$ cycle. Distinctively, the battery of hybrid electrolyte without BN addition only shows capacities of below 125.0 $\mathrm{mA} \mathrm{h} \mathrm{g}{ }^{-1}$ and much faster degradation. The charge-discharge voltage profiles (between 2.5 and $4.0 \mathrm{~V}$ ) of the battery of hybrid electrolyte with $1 \% \mathrm{BN}$ are displayed in Fig. $5 \mathrm{~d}$, which are obtained from the $1^{\text {st }}, 2^{\text {nd }}, 3^{\text {rd }}, 10^{\text {th }}$ and $50^{\text {th }}$ cycles. The reaction plateaus are well corresponding to the characterization of $\mathrm{LiFePO}_{4}$ cathode, which remains with the same pattern, indicating excellent electrochemical stability of the hybrid electrolyte during the 50 cycles without remarkable polarization.[16] It is noteworthy that for the first charge, the battery with liquid electrolyte delivers a much higher capacity of $293.8 \mathrm{~mA} \mathrm{~h} \mathrm{~g}^{-1}$, almost double the values of those of hybrid electrolytes (154.5 $\mathrm{mA} \mathrm{h} \mathrm{g}^{-1}$ for the battery with $1 \% \mathrm{BN}$ in the hybrid electrolyte). Therefore, the initial Coulombic efficiency of the batteries are 50\%, 90\%, 97.6\% and $96.8 \%$ for the batteries with liquid electrolyte, hybrid electrolytes without $\mathrm{BN}$, with $1 \% \mathrm{BN}$ and $10 \% \mathrm{BN}$ addition, respectively. During the subsequent 50 cycles, the battery of hybrid electrolyte with $1 \%$ BN exhibits the highest Coulombic efficiency of $98.1 \%$ in average.

Ionic conductivities of liquid electrolyte $\left(1 \mathrm{M} \mathrm{LiPF}_{6}\right.$ in EC/DMC $\left.1: 1\right)$ and the hybrid electrolyte with $1 \% \mathrm{BN}$ are $1-10 \times 10^{-3} \mathrm{~S} \mathrm{~cm}^{-1}$ and $1.82 \times 10^{-3} \mathrm{~S} \mathrm{~cm}^{-1}$, respectively. Due to the contact interface is much larger between electrode and liquid electrolyte than that between electrode and hybrid electrolyte, the battery with liquid electrolyte show much lower impedance than solid electrolyte. At lower rate, the capacity of cathode is less dominated by the ion mobility, so the battery capacities of liquid electrolyte and hybrid electrolyte with $1 \% \mathrm{BN}$ at lower rate (like $0.1 \mathrm{C}$ and $0.2 \mathrm{C}$ ) are similar. However, at higher current densities, the capacities are more significantly affected by the ionic transport, especially the interfacial resistance between electrode and electrolyte. So the rate performance of liquid electrolyte is superior to that of hybrid electrolytes (Fig. 5b). As compared with 
liquid electrolyte, the hybrid electrolyte with $1 \%$ BN has less intimate contact between electrode and electrolyte, thus exhibits a higher overall resistance, but worse cycling performance at high current density $(1 \mathrm{C}$, as shown in Fig. S13). The unsatisfied rate performance and cycling performance of the hybrid electrolyte with $1 \% \mathrm{BN}$ at higher current density is considered to be due to the small contact interface between electrode and electrolyte (only surface contact of about $1.32 \mathrm{~cm}^{2}$, much smaller than that of liquid electrolyte), which is significantly affected by the electrode, especially with a high mass loading cathode $\left(13.6 \mathrm{mg} \mathrm{cm}^{-2}\right)$. However, at a low current density $(0.2 \mathrm{C})$, the hybrid electrolyte with $1 \% \mathrm{BN}$ shows superior cycling performance and Coulombic efficiency (Fig. 5c and 5e). It is considered that the very low initial Coulombic efficiency (50\%) of the battery with liquid electrolyte is due to the excess SEI formation on cathode material surface, while the SEI formation is not so prominent with the hybrid electrolyte thus inducing higher initial Coulobmic efficiency in the batteries with hybrid electrolyte.[40] In summary, the polymer/salt hybrid electrolyte with $1 \%$ BN exhibits better performance than the batteries of hybrid electrolytes without $\mathrm{BN}$ or with $10 \% \mathrm{BN}$, and even better than that of the liquid electrolyte due to the high ionic conductivity, enhanced mechanical strength, and electrochemical stability. 

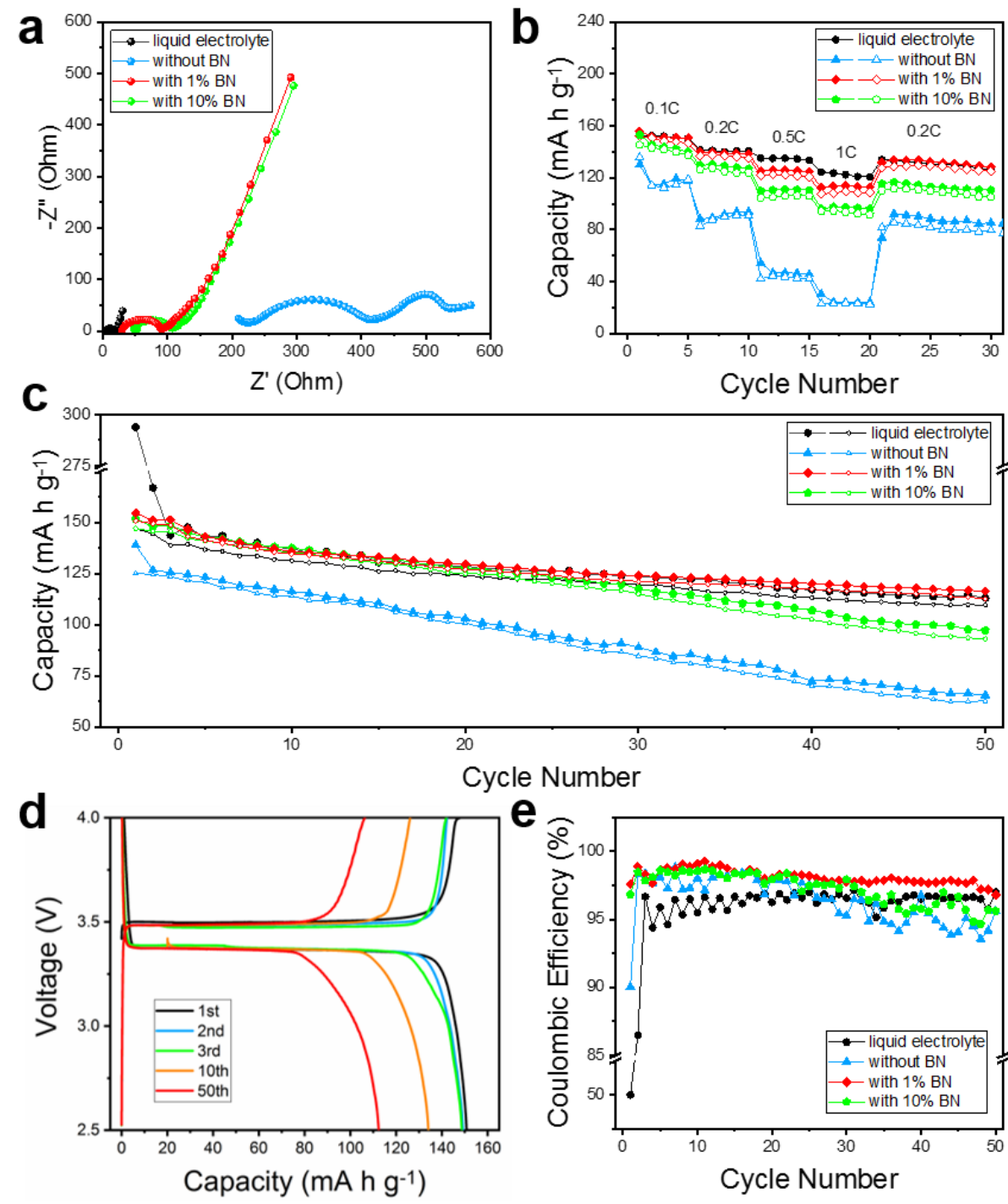

Fig. 5. For the liquid electrolyte and polymer/salt hybrid electrolytes without BN, with $1 \%$ BN and 10\% BN: (a) EIS of asymmetric batteries with LFP cathode. (b) rating performance of batteries with current rates of $0.1 \mathrm{C}, 0.2 \mathrm{C}$, $0.5 \mathrm{C}$ and $1 \mathrm{C}$. (c) cycling performance at $0.2 \mathrm{C}$ for 50 cycles. (d) charge-discharge profiles of the $1^{\text {st }}, 2^{\text {nd }}, 3^{\text {rd }}, 10^{\text {th }}$, and $50^{\text {th }}$ cycles of the battery using hybrid electrolyte with $1 \%$ BN. (e) Coulombic efficiency of the batteries during the 50 cycles.

\section{Conclusions}

We have successfully fabricated a polymer/salt (PVDF-HFP/LiTFSI) hybrid electrolyte for all-solid-state LIBs via a novel and facile method, which was demonstrated to exhibit improved properties with $1 \% \mathrm{BN}$ addition. The optimized hybrid electrolyte with BN addition was enhanced with a higher ionic conductivity, improved mechanical strength and better interfacial contact between the electrolyte and electrodes. As a result, the hybrid electrolyte was demonstrated to effectively suppress the lithium dendrite growth during striping and plating. 
Subsequently, battery composed of lithium metal anode and $\mathrm{LiFePO}_{4}$ cathode using the as-fabricated $1 \% \mathrm{BN}$ enhanced polymer/salt hybrid electrolyte exhibited improved cycling performance $\left(116 \mathrm{~mA} \mathrm{~h} \mathrm{~g}^{-1}\right.$ after 50 cycles at $0.2 \mathrm{C}$ ) with high Coulombic efficiency (over 98\%). We believe the present work provide a facile process for a high-performance electrolyte of all-solid-state LIBs with high energy density, as well as satisfied reliability.

\section{Acknowledgements}

All the experiments were carried out at the UCL Institute for Materials Discovery.

\section{Appendix A. Supplementary data}

Supplementary data to this article can be found online at ...

\section{References}

[1] S. Chandrashekar, N.M. Trease, H.J. Chang, L.S. Du, C.P. Grey, A. Jerschow, ${ }^{7}$ Li MRI of Li batteries reveals location of microstructural lithium, Nat. Mater. 11 (2012) 311-315.

[2] J.C. Bachman, S. Muy, A. Grimaud, H.H. Chang, N. Pour, S.F. Lux, O. Paschos, F. Maglia, S. Lupart, P. Lamp, L. Giordano, S.H. Yang, Inorganic solid-state electrolytes for lithium batteries: mechanisms and properties governing ion conduction, Chem. Rev. 116 (2016) 140-162.

[3] Z.H. Gao, H.B. Sun, L. Fu, F.L. Ye, Y. Zhang, W. Luo, Y.H. Huang, Properties, challenges, and recent progress of inorganic solid-state electrolytes for all-solid-state lithium batteries, Adv. Mater. 30 (2018) 1705702.

[4] Y. Kato, S. Hori, T. Saito, K. Suzuki, M. Hirayama, A. Mitsui, M. Yonemura, H. Iba, R. Kanno, High-power all-solid-state batteries using sulphide superionic conductors, Nat. Energy 1 (2016) 16030.

[5] N. Kamaya, K. Homma, Y.Yamakawa, M. Hirayama, R. Kanno, M. Yonemura, T. Kamiyama, Y. Kato, S. Hama, K. Kawamoto, A. Mitsui, A lithium superionic conductor, Nat. Mater. 10 (2011) 682-686.

[6] C.W. Sun, J. Liu, Y.D. Gong, D.P. Wilkinson, J.J. Zhang, Recent advances in all-solid-state rechargeable lithium batteries, Nano Energy 33 (2017) 363-386.

[7] S.H. Yu, R.D. Schmidt, R. Garcia-Mendez, E. Herbert, N.J. Dudney, J.B. Wolfenstine, J. Sakamoto, D.J. Siegel, Elastic properties of the solid electrolyte $\mathrm{Li}_{7} \mathrm{La}_{3} \mathrm{Zr}_{2} \mathrm{O}_{12}$ (LLZO), Chem. Mater. 28 (2016), 197-206. 
[8] L.Z. Long, S.J. Wang, M. Xiao, Y.Z. Meng, Polymer electrolytes for lithium polymer batteries, J. Mater. Chem. A 4 (2016) 10038-10069.

[9] C.W. Nan, L.Z. Fan, Y.H. Lin, Q. Cai, Enhanced ionic conductivity of polymer electrolytes containing nanocomposite $\mathrm{SiO}_{2}$ particles, Phys. Rev. Lett. 91 (2003) 266104.

[10] R. Khurana, J.L. Schaefer, L.A. Archer, G.W. Coates, Suppression of lithium dendrite growth using crosslinked polyethylene/poly(ethylene oxide) electrolytes: a new approach for practical lithium-metal polymer batteries, J. Am. Chem. Soc. 136 (2014) 7395-7402.

[11] H.P. Wu, Y. Cao, H.P. Su, C. Wang, Tough gel electrolyte using double polymer network design for the safe, stable cycling of lithium metal anode, Angew. Chem. Int. Ed. 57 (2018) 1361-1365.

[12] L. Chen, L.Z. Fan, Dendrite-free Li metal deposition in all-solid-state lithium sulphur batteries with polymerin-salt polysiloxane electrolyte, Energy Storage Mater. 15 (2018), 37-45.

[13] S. Choudhury, R. Mangal, A. Agrawal, L.A. Archer, A highly reversible room-temperature lithium metal battery based on crosslinked hairy nanoparticles, Nat. Commun.6 (2015) 10101.

[14] L. Chen, Y.T. Li, S.P. Li, L.Z. Fan, C.W. Nan, J.B. Goodenough, PEO/garnet composite electrolytes for solid-state lithium batteries: from “ceramic-in-polymer" to "polymer-in-ceramic”. Nano Energy 46 (2018) 176184.

[15] J.Y. Song, Y.Y. Wang, C.C. Wan, Review of gel-type polymer electrolytes for lithium-ion batteries, J. Power Sources 77 (1999) 183-197.

[16] J. Shim, H.J. Kim, B.G. Kim, Y.S. Kim, D.G. Kim, J.C. Lee, 2D boron nitride nanoflakes as a multifunctional additive in gel polymer electrolytes for safe, long cycle life and high rate lithium metal batteries, Energy Environ. Sci. 10 (2017) 1911-1916.

[17] K.H. Oh, D. Lee, M.J. Choo, K.H. Park, S. Jeon, S.H. Hong, J.K. Park, J.W. Choi, Enhanced durability of polymer electrolyte membrane fuel cells by functionalize 2D boron nitride nanoflakes, ACS Appl. Mater. Inter. 6 (2014) 7751-7758.

[18] M.T.F. Rodrigues, K. Kalaga, H. Gullapalli, G. Babu, A.L.M. Reddy, P.M. Ajayan, Hexagonal boron nitridebased electrolyte composite for Li-ion battery operation from room temperature to $150{ }^{\circ} \mathrm{C}$, Adv. Energy Mater. 6 (2016) 1600218. 
[19] L. Shi, A. Xu, T.S. Zhao, First-principles investigations of the working mechanism of 2D h-BN as an interfacial layer for the anode of lithium metal batteries, ACS Appl. Mater. Inter. 9 (2017) 1987-1994.

[20] S.H. Yeon, K.S. Kim, S. Choi, J.H. Cha, H. Lee, Characterization of PVdF(HFP) gel electrolytes based on 1-(2-Hydroxyethyl)-3-methyl imidazolium ionic liquids. J. Phys. Chem. B 109 (2005) 17928-17935.

[21] S. Ramesh, O.P. Ling, Effect of ethylene carbonate on the ionic conduction in poly(vinylidenefluoridehexafluoropropylene) based solid polymer electrolytes, Polym. Chem. 1 (2010) 702-707.

[22] N. Aliahmad, S. Shrestha, K. Varahramyan, M. Agarwal, Poly(vinylidene fluoride-hexafluoropropylene) polymer electrolyte for paper-based and flexible battery applications, AIP Adv. 6 (2016) 065206.

[23] N. N. Saadun, R. Subramaniam, R. Kasi, Development and characterization of poly(1-vinylpyrrolidone-covinyl acetate) copolymer based polymer electrolytes, Sci. World J. (2014) 254215.

[24] P.M. Sudeep, S. Vinod, S. Ozden, R. Sruthi, A. Kukovecz, Z. Konya, R. Vajtai, M.R. Anantharaman, P.M. Ajayan, T.N. Narayanan, Functionalized boron nitride porous solids, RSC. Adv. 5 (2015) 93964.

[25] N. Ataollahi, A. Ahmad, H. Hamzah, M.Y. A. Rahman, N.S. Mohamed, Preparation and characterization of PVDF-HFP/MG49 based polymer blend electrolyte, Int. J. Electrochem. Sci. 7 (2012) 6693-6703.

[26] Z. D. Liu, Y. Feng, W. L. Li, High dielectric constant and low loss of polymeric dielectric composites filled by carbon nanotubes adhering $\mathrm{BaTiO}_{3}$ hybrid particles, RSC Adv. 5 (2015) 29017-29021.

[27] M. Kerner, N. Plylahan, J. Scheers, P. Johanson, Ionic liquid based lithium battery electrolytes: fundamental benefits of utilising both TFSI and FSI anions? Phys. Chem. Chem. Phys. 17 (2015) 19569-19581.

[28] Shalu; V.K. Singh, R.K. Singh, Development of ion conducting polymer gel electrolyte membranes based on polymer PVdF-HFP, BMIMTFSI ionic liquid and the Li-salt with improved electrical, thermal and structural properties. J. Mater. Chem. C 3 (2015) 7305-7318.

[29] J. Amici, M. Alidoost, C. Francia, S. Bodoardo, S.M. Crespiera, D. Amantia, M. Biasizzo, F. Caldera, F. Trotta, $\mathrm{O}_{2}$ selective membranes base on a dextrin-nanosponge (NS) in a PVDF-HFP polymer matrix for Li-air cells. Chem. Commun. 52 (2016) 13683-13686.

[30] K. Yang, X.Y. Huang, L.J. Fang, J.L. He, P.K. Jiang, Fluoro-polymer functionalized graphene for flexible ferroelectric polymer-based high-k nanocomposites with suppressed dielectric loss and low percolation threshold. Nanoscale 6 (2014) 14740-14753. 
[31] X. Zhang, T. Liu, S.F. Zhang, X. Huang, B.Q. Xu, Y.H. Lin, B. Xu, L.L. Li, C.W. Nan, Y. Shen, Synergistic coupling between $\mathrm{Li}_{6.25} \mathrm{La}_{3} \mathrm{Zr}_{1.75} \mathrm{Ta}_{0.25} \mathrm{O}_{12}$ and poly(vinylidene fluoride) induces high ionic conductivity, mechanical strength, and thermal stability of solid composite electrolytes, J. Am. Chem. Soc. 139 (2017) 1377913785.

[32] P.M. Shanthi, P.J. Hanumantha, T. Albuquerque, B. Gattu, P.N. Kumta, Novel composite polymer electrolytes of PVdF-HFP derived by electrospinning with enhanced Li-ion conductivities for rechargeable lithium-sulfur batteries, ACS Appl. Energy Mater. 1 (2018) 483-494.

[33] J.M. Tarascon, A.S. Gozdz, C. Shmutz, F. Shokoohi, P.C. Warren, Performance of bellcore's plastic rechargeable Li-ion batteries. Solid State Ionics 86-88(1996) 49-54.

[34] X.K. Zhang, J. Xie, F.F. Shi, D.C. Lin, Y.Y. Liu, W. Liu, A. Pei, Y.J. Gong, H.X. Wang, K. Liu, Y. Xiang, Y. Cui, Vertically aligned and continuous nanoscale ceramic-polymer interfaces in composite solid polymer electrolytes for enhanced ionic conductivity, Nano Lett. 18 (2018) 3829-3838.

[35] S. Das, A. Ghosh, Ion conduction and relaxation in PEO-LiTFSI- $\mathrm{Al}_{2} \mathrm{O}_{3}$ polymer nanocomposite electrolytes, J. Appl. Phys. 117 (2015) 174103.

[36] C. C. Tambelli, A. C. Bloise, A. V. Rosario, E. C. Pereira, C. J. Magon, J. P. Donoso, Characterisation of PEO- $\mathrm{Al}_{2} \mathrm{O}_{3}$ composite polymer electrolytes, Electrochim. Acta 47 (2002) 1677-1682.

[37] P.C. Yao, B. Zhu, H.W. Zhai, X.B. Liao, Y.X. Zhu, W.H. Xu, Q. Cheng, C. Jayyosi, Z. Li, J. Zhu, K.M. Myers, X. Chen, Y. Yang, PVDF/Palygorshite nanowire composite electrolyte for 4V rechargeable lithium batteries with high energy density, Nano Lett. 18 (2018), 6113-6120.

[38] J. Bae, Y. T. Li, F. Zhao, X. Y. Zhou, Y. Ding, G. H. Yu, Designing 3D nanostructured garnet frameworks for enhancing ionic conductivity and flexibility in composite polymer electrolytes for lithium batteries, Energy Storage Mater. 15 (2018) 46-52.

[39] K. Fu, Y.H. Gong, J.Q. Dai, A. Gong, X.G. Han, Y.G. Yao, C.W. Wang, Y.B. Wang, Y.N. Chen, C.Y. Yan, Y.J. Li, E.D. Wachsman, L.B. Hu, Flexible, solid-state, ion-conducting membrane with 3D garnet nanofiber networks for lithium batteries, P. Natl. Acad. Sci. USA 113 (2016) 7094-7099. 
[40] J. J. Zhang, J. F. Yang, T. T. Dong, M. Zhang, J. C. Chai, S. M. Dong, T. Y. Wu, X. H. Zhou, G. L. Cui, Aliphatic polycarbonate-based solid-state polymer electrolytes for advanced lithium batteries: advances and perspective, Small 2018, 14, 1800821. 